\title{
Oral Vaccination of Grass Carp (Ctenopharyngodon idella) with Baculovirus-Expressed Grass Carp Reovirus (GCRV) Proteins Induces Protective Immunity against GCRV Infection
}

\author{
Changyong Mu ${ }^{1,2,+}$, Qiwang Zhong ${ }^{2,+}$, Yan Meng ${ }^{1}$, Yong Zhou ${ }^{1}$, Nan Jiang ${ }^{1}$, Wenzhi Liu ${ }^{1}$, Yiqun Li ${ }^{1}$, \\ Mingyang Xue ${ }^{1}$, Lingbing Zeng ${ }^{1}$, Vikram N. Vakharia ${ }^{3, *(D)}$ and Yuding Fan ${ }^{1,2,3,4, *}$
}

1 Yangtze River Fisheries Research Institute, Chinese Academy of Fishery Sciences, Wuhan 430223, China; muchangyong@yfi.ac.cn (C.M.); mengy@yfi.ac.cn (Y.M.); zhouy@yfi.ac.cn (Y.Z.); jn851027@yfi.ac.cn (N.J.); liuwenzhialisa@yfi.ac.cn (W.L.); liyq@yfi.ac.cn (Y.L.); xmy@yfi.ac.cn (M.X.); zlb@yfi.ac.cn (L.Z.)

2 College of Biological Science and Engineering, Jiangxi Agricultural University, Nanchang 330045, China; zhongqiwang@jxau.edu.cn

3 Institute of Marine and Environmental Technology, University of Maryland Baltimore Country, Baltimore, MD 21202, USA

4 State Key Laboratory of Developmental Biology of Freshwater Fish, College of Life Sciences, Hunan Normal University, Changsha 410081, China

* Correspondence: vakharia@umbc.edu (V.N.V.); fanyd@yfi.ac.cn (Y.F.)

+ These two authors contributed equally to this work.

Citation: Mu, C.; Zhong, Q.;

Meng, Y.; Zhou, Y.; Jiang, N.; Liu, W.; Li, Y.; Xue, M.; Zeng, L.; Vakharia,

V.N.; Fan, Y.et al. Oral Vaccination of Grass Carp (Ctenopharyngodon idella) with Baculovirus-Expressed Grass Carp Reovirus (GCRV) Proteins Induces Protective Immunity against GCRV Infection. Vaccines 2021, 9, 41. https://doi.org/10.3390/ vaccines 9010041

Received: 29 November 2020

Accepted: 10 January 2021

Published: 12 January 2021

Publisher's Note: MDPI stays neutral with regard to jurisdictional clai$\mathrm{ms}$ in published maps and institutional affiliations.

Copyright: (C) 2021 by the authors. Licensee MDPI, Basel, Switzerland. This article is an open access article distributed under the terms and conditions of the Creative Commons Attribution (CC BY) license (https:// creativecommons.org/licenses/by/ $4.0 /)$.
Abstract: The grass carp reovirus (GCRV) causes severe hemorrhagic disease with high mortality and leads to serious economic losses in the grass carp (Ctenopharyngodon idella) industry in China. Oral vaccine has been proven to be an effective method to provide protection against fish viruses. In this study, a recombinant baculovirus BmNPV-VP35-VP4 was generated to express VP35 and VP4 proteins from GCRV type II via Bac-to-Bac baculovirus expression system. The expression of recombinant VP35-VP4 protein (rVP35-VP4) in Bombyx mori embryo cells (BmE) and silkworm pupae was confirmed by Western blotting and immunofluorescence assay (IFA) after infection with BmNPV-VP35-VP4. To vaccinate the grass carp by oral route, the silkworm pupae expressing the rVP35-VP4 proteins were converted into a powder after freeze-drying, added to artificial feed at $5 \%$ and fed to grass carp (18 $\pm 1.5 \mathrm{~g})$ for six weeks, and the immune response and protective efficacy in grass carp after oral vaccination trial was thoroughly investigated. This included blood cell counting and classification, serum antibody titer detection, immune-related gene expression and the relative percent survival rate in immunized grass carp. The results of blood cell counts show that the number of white blood cells in the peripheral blood of immunized grass carp increased significantly from 14 to 28 days post-immunization (dpi). The differential leukocyte count of neutrophils and monocytes were significantly higher than those in the control group at $14 \mathrm{dpi}$. Additionally, the number of lymphocytes increased significantly and reached a peak at $28 \mathrm{dpi}$. The serum antibody levels were significantly increased at Day 14 and continued until 42 days post-vaccination. The mRNA expression levels of immune-related genes (IFN-1, TLR22, IL-1 $\beta$, MHC I, Mx and IgM) were significantly upregulated in liver, spleen, kidney and hindgut after immunization. Four weeks postimmunization, fish were challenged with virulent GCRV by intraperitoneal injection. The results of this challenge study show that orally immunized group exhibited a survival rate of $60 \%$ and relative percent survival (RPS) of 56\%, whereas the control group had a survival rate of $13 \%$ and RPS of $4 \%$. Taken together, our results demonstrate that the silkworm pupae powder containing baculovirusexpressed VP35-VP4 proteins could induce both non-specific and specific immune responses and protect grass carp against GCRV infection, suggesting it could be used as an oral vaccine.

Keywords: grass carp reovirus (GCRV); baculovirus expression; oral vaccine; immune response 


\section{Introduction}

Grass carp (Ctenopharyngodon idella) is an economically important freshwater fish, occupying a vital position in aquaculture of China [1]. However, hemorrhagic disease caused by grass carp reovirus (GCRV) poses a serious threat to the grass carp cultivation industry [2], resulting in a greater than $80 \%$ mortality rate and significant economic losses [3] GCRV, a member of the genus Aquareovirus in the family Reoviridae, was the first viral pathogen identified from aquatic animals in China in 1983 [4]. A comparative study of gene sequences revealed that GCRV could be divided into three distinct genotypes: GCRV I with GCRV-873 as the representative strain, GCRV II with GCRV-HZ08 as the representative strain and GCRV III with GCRV-104 being the only strain found in China [5]. Epidemiological analyses have shown that the three genotypes exist simultaneously, but GCRV II accounts for the major pandemic of grass carp hemorrhagic disease in China [6]. Therefore, it is necessary to find an efficacious novel vaccine for preventing the hemorrhagic disease caused by GCRV II. At present, some vaccines, including inactivated vaccines, attenuated vaccines, recombinant subunit vaccines and DNA vaccines, have been developed and widely used to protect grass carp. However, the variations of different GCRV strains have led to the need of effective subunit vaccines, which can be developed by recombinant techniques [7].

Virus structural proteins often serve as a key antigen capable of stimulating potent immune response against viral infections [8]. To date, several GCRV structural proteins, such as VP35, VP4, VP6 and VP56, have been investigated as the potential subunit vaccine against GCRV infection. For example, GCRV-VP56 expressed in Escherichia coli induced significant immuno-protective effects and provided a protective response of about $71-75 \%$ for injection-vaccination in grass carp [9]. In another study, Xue et al. (2013) showed that the silkworm pupae powder containing baculovirus-expressed VP6 protein administered orally with feed induced BacFish-vp6 specific antibody in grass carp [10]. VP4 protein is the major outer capsid protein encoded by GCRV segment 6 (S6) and can be used as a candidate subunit vaccine [3], which plays an important role in viral invasion and replication [11,12]. Bioinformatics analysis predicted that the VP35 protein encoded by segment 11 (S11) of GCRV II contained a conserved putative zinc-binding motif CxxC-n16-HxC sequence and was considered to be an outer clamp protein [13]. Liu et al. showed that anti-VP35 serum could effectively neutralize GCRV infection [14] and Gao et al. suggested that recombinant VP35 protein can induce immunity and protect grass carp against GCRV infection [7]. Therefore, the VP35 protein is predicted to be an outer capsid protein and has antigenicity, and hence can be used as a subunit vaccine [7].

The baculovirus expression system has been widely employed as a powerful expression vector for the production of recombinant proteins and development of subunit vaccines in insect cells due to its biological safety, limited replication in insect cells, low cytotoxicity and simplicity of operation $[15,16]$. Baculoviruses are double-stranded DNA viruses known to infect invertebrates, of which Autographa californica nucleopolyhedrovirus (AcMNPV) and Bombyx mori nucleopolyhedrovirus (BmNPV) are the most widely studied [17]. Moreover, the silkworm baculovirus expression system has a potential for not only low-cost but also high-capacity production (up to $20 \%$ of total cell protein), which is an ideal system for producing vaccines [10].

The oral vaccination route has the advantages of being inexpensive, time-saving and easy to administer without causing any stress to the fish $[10,18]$. In the present study, we generated a novel recombinant baculovirus BmNPV-VP35-VP4 expressing the recombinant VP35-VP4 protein (rVP35-VP4) of GCRV II and used it to infect silkworm pupae to prepare a lyophilized powder for oral immunity to grass carp. We determined the immune response and further evaluated the protective effects of the recombinant VP35-VP4 protein against GCRV infection in grass carp. These results will help us to understand the immune protective mechanism of viral protein and lay a foundation for the development of oral vaccine for GCRV. 


\section{Materials and Methods}

\subsection{Ethics Statement}

The experiment was carried out in strict accordance with the Guide for the Care and Use of Laboratory Animals Monitoring Committee of Hubei Province, China, and the internal protocols (No. YFI2019fanyuding-02) were approved by the Committee on the Ethics of Animal Experiments at the Yangtze River Fisheries Research Institute, Chinese Academy of Fishery Sciences.

\subsection{Fish, Virus, Cells and Plasmid}

Healthy grass carp, with an average weight of $18 \pm 1.5 \mathrm{~g}$, were obtained from a farm in Wuhan City (Hubei, China), which were acclimatized to laboratory conditions for two weeks before experimental manipulation. Fish were maintained at $28^{\circ} \mathrm{C}$ in aerated water and fed twice a day. From this pool, fish were randomly tested for the presence of GCRV by RT-PCR to ensure that these fish were free of this virus and are not the carriers of GCRV. GCRV-106 strain, used for this study, was isolated from diseased grass carp with severe hemorrhagic disease and identified as GCRV II in our laboratory. Bombyx mori embryo cells were generously provided by $\mathrm{Dr}$. Tian $\mathrm{Li}$, Southwest University, China, and maintained at $28{ }^{\circ} \mathrm{C}$ in Grace's Insect Medium containing 10\% (v/v) FBS (Gibco, Gaithersburg, MD, USA). Cell transfection reagent Cellfectin ${ }^{\mathrm{TM}}$ II Reagent was purchased from Thermo Fisher (Waltham, MA, USA). The pFastBac-VP35-T2A-VP4 plasmid was constructed from previous research in our laboratory [19].

\subsection{Generation of Recombinant Baculovirus}

According to the manufacturer's instructions, the pFastBac-VP35-T2A-VP4 vector was transformed into E. coli DH10Bac/BmNPV to generate recombinant Bacmid-VP35VP4, using the Bac-to-Bac baculovirus expression system (Invitrogen, Carlsbad, CA, USA). The recombinant Bacmid-VP35-VP4 is too large (larger than $135 \mathrm{~kb}$ in size) to perform a restriction analysis. Therefore, recombinant Bacmid-VP35-VP4 was confirmed by PCR analysis with the pUC/M13 Forward primer: 5'-CCCAGTCACGACGTTGTAAAACG-3' and Reverse primer: 5'-AGCGGA TAACAATTTCACACAGG-3'. The Bacmid-VP35-VP4 DNA was transfected into BmE cells to generate the recombinant baculovirus BmNPVVP35-VP4 with Cellfectin ${ }^{\mathrm{TM}}$ II transfection reagent (Invitrogen, Carlsbad, CA, USA). The transfected cells were incubated at $28^{\circ} \mathrm{C}$ for $72 \mathrm{~h}$ to generate baculovirus and its release into the culture medium. The culture medium was collected and clarified by low-speed centrifugation for $10 \mathrm{~min}$ at $1000 \times g$ as the P1 virus and the virus continuously proliferated through further infection in BmE cells until the P3 viral stock was obtained and kept at $4{ }^{\circ} \mathrm{C}$ in the dark. The $\mathrm{P} 1$ and $\mathrm{P} 3$ viruses were identified by PCR with primer pairs of GCRV-VP35 F: 5'-GGGATCCATGGAACCAGCAAAACCATG-3' and GCRV-VP4 R: 5' GGAATTCTCTAGTGATGGTGGTGATGATGAGACGGAGGAGGCCAGTATCGAGTTA ATTTGT-3'.

\subsection{Analysis of VP35-VP4 Expression in BmE Cells by Western Blotting}

To confirm that the rVP35-VP4 protein can be expressed in BmE cells, the BmE cells were collected $72 \mathrm{~h}$ after infection with BmNPV-VP35-VP4 for SDS-PAGE and Western blot analysis. BmE cells infected with wild-type BmNPV were used as a negative control. The cell extracts were run on 12\% SDS-PAGE and the separated proteins were transferred onto PVDF membranes. After blocking with 5\% skim milk in phosphate buffered saline (PBS)-Tween (PBST) for $2 \mathrm{~h}$ at $37^{\circ} \mathrm{C}$, the membrane was incubated with a mouse antiHis-tag (1:1000 diluted with TBST, Abcam, Cambridge, UK) and the secondary antibody alkaline phosphatase-conjugated goat anti-mouse IgG (1:5000 diluted with TBST, Thermo Fisher, Waltham, MA, USA) and finally visualized by the enhanced chemiluminescence (ECL) method. 


\subsection{Detection of VP35-VP4 Protein Expression in BmE Cells by IFA}

To identify the rVP35-VP4 protein expression, BmE cells were infected with recombinant BmNPV-VP35-VP4 in a 12-well microplate and incubated for 4 days. The standard indirect IFA was performed according to the method described in our previous study [19].

\subsection{Multiplication and Detection of rVP35-VP4 Protein in Silkworm Pupae}

To proliferate rVP35-VP4 protein in the silkworm, the silkworm on the first day of 5th instar was inoculated by subcutaneous injection with recombinant BmNPV-VP35-VP4 at amount of about $3 \times 10^{4} \mathrm{PFU}$ per silkworm. The silkworm hemolymph was collected at $25{ }^{\circ} \mathrm{C}$ for $120 \mathrm{~h}$ after infection. The negative control silkworm hemolymph was collected at $72 \mathrm{~h}$ post-infection with wild type of BmNPV. All hemolymph samples were subjected to SDS-PAGE and Western blotting according to the method described previously.

\subsection{Oral Immunization and Sample Collection}

Silkworm pupae were collected after $120 \mathrm{~h}$ of inoculation with recombinant virus BmNPV-VP35-VP4 to prepare freeze-dried powder and added to the feed at a rate of $5 \%$. The fish feed containing $5 \%$ freeze-dried powder was made from pupae of inoculation with wild type of BmNPV as a control. Grass carps were randomly divided into three groups ( $n=60$ for each group). The fish in the vaccination group (VP35-VP4) were fed with the feed containing 5\% of freeze-dried powder of the BmNPV-VP35-VP4-infected pupae; the fish in control group (Group CK) were fed with the feed containing $5 \%$ of the freeze-dried powder of the wild type of BmNPV-infected pupae; and the fish in normal group (Group N) were fed with the normal dry pellets. The grass carps were fed with prepared feed continuously in accordance with $2 \%$ of the fish weight, twice a day (in a final concentration equivalent to $0.02 \mu \mathrm{g} / \mathrm{g}$ (protein per fish)) for 6 weeks and the experimental fish were maintained at $25 \pm 2{ }^{\circ} \mathrm{C}$. Thereafter, grass carps in all groups were fed with the normal dry pellets for 4 weeks and the experimental fish were maintained at $25 \pm 2{ }^{\circ} \mathrm{C}$. Blood, head kidney, liver, spleen and hindgut tissues were obtained from three fish in each experimental group at 7, 14, 21, 28, 35 and 42 days post-immunization (dpi). All tissues were placed in DEPC-treated homogenate tube, treated with $1 \mathrm{~mL}$ TRIzol reagent and stored at $-80^{\circ} \mathrm{C}$. Blood sampled from tail venipuncture and the blood cells were counted according to type by using a Neubauer hemocytometer. The blood samples were kept at RT for $2 \mathrm{~h}$ to clot before stored at $4{ }^{\circ} \mathrm{C}$ overnight, followed by centrifuging at $4{ }^{\circ} \mathrm{C}, 1500 \times \mathrm{g}$ for $15 \mathrm{~min}$ to collect serum and stored at $-80^{\circ} \mathrm{C}$. The blood cells were used for the differential leukocyte count of blood smears.

\subsection{Counting of Blood Cells and Differential Leukocytes}

The blood cell counts were done with a 1:200 dilution of the blood sample using Dacie's reagent and counted in a Neubauer hemocytometer [20]. Additionally, the average of triplicate micro-hematocrits was used to determine the number of red blood cells (RBCs) or white blood cells (WBCs) [21]. Triplicate blood smears for each sample were prepared from fresh blood, then blood smears were air-dried, fixed in methanol, stained with Giemsa for $15 \mathrm{~min}$ and washed and dried, and $100 \mathrm{WBC}$ sere randomly counted and classified using oil immersion microscopy [22].

\subsection{Detection of Serum Antibody Titer by Enzyme-Linked Immunosorbent Assay (ELISA)}

According to a previously described method [4], the titer of the antiserum in grass carp was determined by ELISA. Briefly, $100 \mu \mathrm{L}$ stock solutions containing $5 \mu \mathrm{g}$ of rVP35-VP4 was coated in each well in a 96-well plate as antigen. After incubation overnight at $4{ }^{\circ} \mathrm{C}$, it was washed with PBST and blocked with 5\% skimmed milk powder blocking buffer at $37^{\circ} \mathrm{C}$ for $2 \mathrm{~h}$. The plate was washed three times with PBST, and the fish serum (serum was 1:100 dilutions) obtained from the immunized fish at different time points was added to the wells and incubated at $37^{\circ} \mathrm{C}$ for $1 \mathrm{~h}$ as a primary antibody. The plate was washed again and incubated with 1:1000 diluted horseradish peroxidase (HRP) conjugated rabbit 
anti-grass carp IgM antibody at $37^{\circ} \mathrm{C}$ for $30 \mathrm{~min}$ as a secondary antibody. The dilution of the antibody was according to the previous method and product specification $[7,9]$. Plates

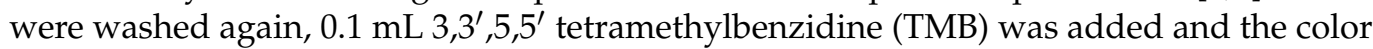
was developed for $30 \mathrm{~min}$ at room temperature. The reaction was stopped by adding $100 \mu \mathrm{L}$ of $2 \mathrm{M}$ sulfuric acid. After calibration with blank control, the $\mathrm{OD}_{450} \mathrm{~nm}$ of samples was read by automated microtiter plate reader.

\subsection{Determination of Immune-Related Genes Expression by qRT-PCR}

Total RNA was extracted by TRIzol regent (Invitrogen, Carlsbad, CA, USA) in accordance with the manufacturer's instructions. First strand cDNA was synthesized using the PrimeScript ${ }^{\mathrm{TM}} 1$ st strand cDNA Synthesis Kit (TaKaRa, Dalian, China). The expression of genes involved in the immune response (IFN-1, TLR22, IL-1 $\beta$, MHC I, Mx and IgM) were determined by quantitative real-time polymerase chain reaction (qRT-PCR). The primers for each gene are listed in Table 1. All qRT-PCRs were performed using TB Green ${ }^{\circledR}$ Premix Ex Taq ${ }^{\mathrm{TM}}$ II (TaKaRa) and the amplification conditions were carried out as described in our previous study [19]. All samples from the immunized and the control groups were tested in triplicate by using $\mathrm{qPCR}$. The gene expression was normalized using the housekeeping gene $\beta$-actin. The relative expression ratios of immune-related genes were analyzed by the $2^{-\Delta \Delta \mathrm{CT}}$ method [23].

Table 1. Primers used for real-time PCR analysis.

\begin{tabular}{|c|c|c|c|c|}
\hline Gene & Primers $\left(5^{\prime}-3^{\prime}\right)$ & Annealing Temperature $\left({ }^{\circ} \mathrm{C}\right)$ & Product Size (bp) & Accession No. \\
\hline TLR22 & $\begin{array}{l}\text { F:CCATCCATTTAACAGGTGCATAC } \\
\text { R:CAGCAGATGTGGAAAGAGACC }\end{array}$ & 58 & 174 & JQ670915.1 \\
\hline IL-1 $\beta$ & $\begin{array}{l}\text { F:TGTGACGCTGAGAGACGGAAA } \\
\text { R:GAGTTTCAGTGACCTCCTTCAA }\end{array}$ & 60 & 190 & JX014320 \\
\hline IgM & $\begin{array}{l}\text { F:GAGGCATCGGAGGCACATTTC } \\
\text { R:TTGGGTCTCGCACCATTTTCTC }\end{array}$ & 55 & 166 & DQ417927 \\
\hline Mx1 & $\begin{array}{l}\text { F:CTGGGGAGGAAGTAAAGTGTTCT } \\
\text { R:CAGCATGGATTCTGCCTGG }\end{array}$ & 57 & 391 & HQ245104 \\
\hline IFN-I & $\begin{array}{c}\text { F:AAGCAACGAGTCTTTGAGCCT } \\
\text { R:GCGTCCTGGAAATGACACCT }\end{array}$ & 58 & 78 & DQ357216 \\
\hline MHC I & $\begin{array}{l}\text { F:CCTGGCAGAAAAATGGACAAG } \\
\text { R:CCAACAACACCAATGACAATC }\end{array}$ & 56 & 271 & AY391782 \\
\hline$\beta$-actin & $\begin{array}{l}\text { F:GATGATGAAATTGCCGCACTG } \\
\text { R:TGGTCAGCCCGAAACTATC }\end{array}$ & 58 & 151 & M25013 \\
\hline
\end{tabular}

\subsection{Challenge Test}

All 30 fish in each group were intraperitoneally injected with $10 \mu \mathrm{L}$ of $1 \times 10^{5} \mathrm{LD}_{50}$ GCRV-106 on 56 days post immunization and then the mortality was recorded daily. The dead fish were collected and RNA from the spleen was extracted to detect the presence of the virus using PCR. The relative percent survival (RPS) was calculated after 14 days of post infection by the following formula: RPS $=(1-$ the ratio of mortality percent in the immunized group to in the control group) $\times 100 \%$.

\subsection{Statistical Analysis}

The results were expressed as the mean \pm standard deviation (SD) and the statistical analysis were carried out with SPSS 20.0 software (SPSS Inc., Chicago, IL, USA). Comparisons between the experimental group and the control group were analyzed by using ANOVA and Tukey's tests and differences were defined as statistically significant at $p<0.05$ and extremely significant at $p<0.01$. 


\section{Results}

\subsection{Generation of Recombinant Baculoviruses in BmE Cells}

To identify the recombinant bacmid, PCR was performed with primers M13 forward and M13 reverse (Figure 1A). A $5.2 \mathrm{~kb}$ product was obtained, which was consistent with the theoretical molecular weight detected, suggesting the VP35-VP4 expression cassettes had integrated into the bacmid genomic DNA (Figure 1B). Furthermore, the recombinant plasmid (Bacmid-VP35-VP4) was verified as being correct by sequencing. The purified Bacmid-VP35-VP4 DNA was transfected into BmE cells to obtain recombinant baculovirus, and the transfected BmE cells showed enlarged cell diameter and nuclei sizes, granular appearance, cessation of cell growth, detachment from the flask and cell lysis at three days post-transfection (Figure 2). After three cycles of infection, high titer P3 virus was used as a template and confirmed using PCR with the primers GCRV-VP35/GCRV-VP4 (Figure 3), suggesting that recombinant baculovirus BmNPV-VP35-VP4 was successfully generated.

A

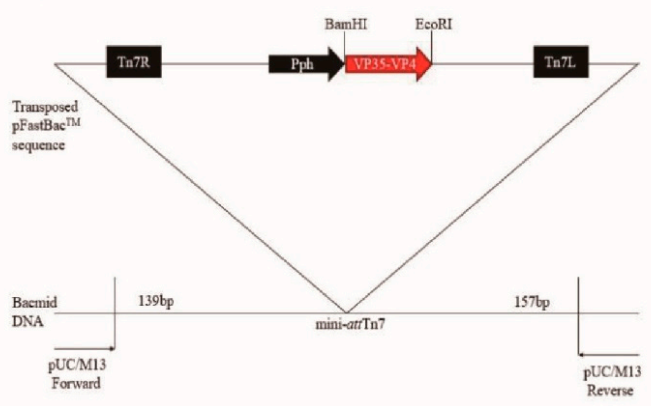

B

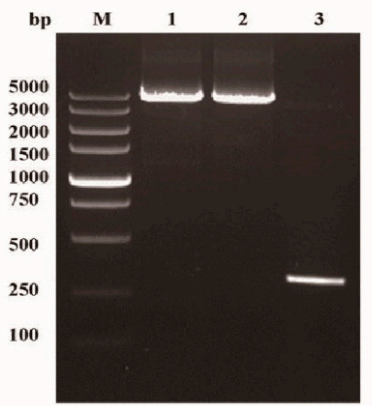

Figure 1. Construction and identification of the recombinant BmBacmid-VP35-VP4: (A) schematic construction of the recombinant baculovirus BmNPV-VP35-VP4; and (B) PCR amplification of VP35VP4 from BmBacmid-VP35-VP4 (Lane M, DNA marker; Lanes 1-2, Bacmid-VP35-VP4; Lane 3, Bacmid alone).

A

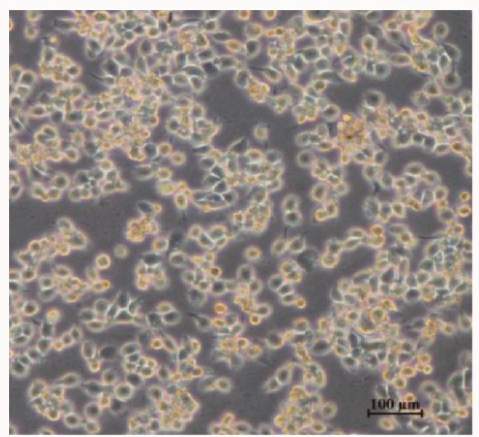

C

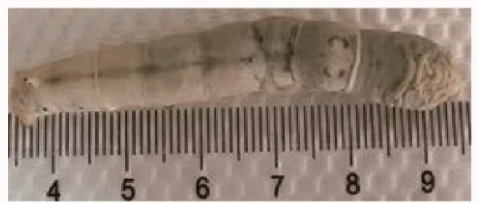

$\mathrm{E}$

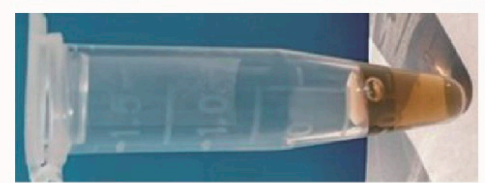

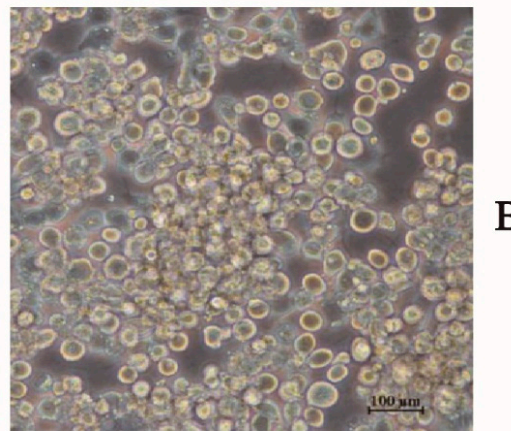

B
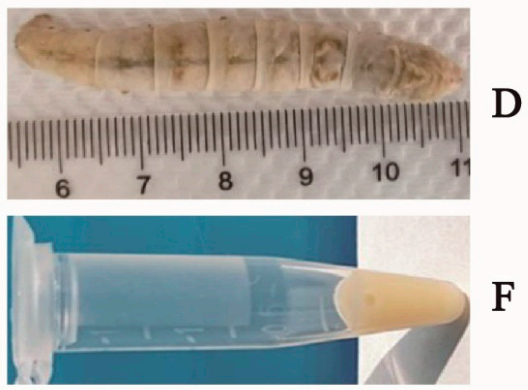

Figure 2. Symptoms of BmE cells and silkworms after transfected/infected with recombinant baculovirus: (A) normal BmE cells; (B) BmE cells transfected with recombinant BmBacmid-VP35-VP4; (C) normal silkworm; (D) silkworm infected with rBmBac-VP35-VP4; (E) hemolymph of normal silkworm; and (F) hemolymph of silkworm infected with rBmBac-VP35-VP4. 

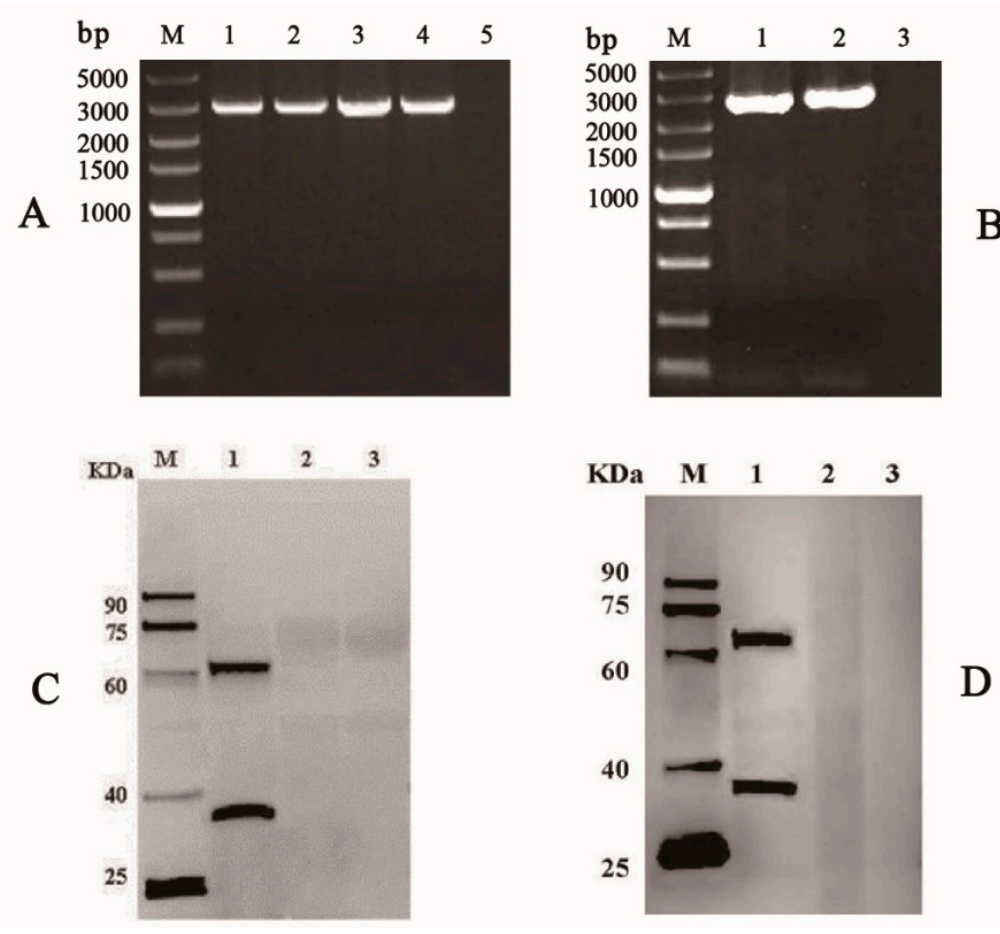

Figure 3. Confirmation and expression analysis of VP35-VP4 in BmE cells and hemolymph of silkworms by PCR and Western blotting. (A) PCR amplification of VP35-VP4 from the genome DNA of P1, P2, and P3 recombinant baculoviruses in BmE cells: Lane M, DNA marker; Lanes 1-3, P1-P3 rBmBac-VP35-VP4; Lane 4, rBmBacmid-VP35-VP4; Lane 5, rBmBac-wild. (B) PCR amplification of VP35-VP4 in hemolymph of silkworms: Lane M, DNA marker; Lane 1, Hemolymph of silkworms infected with rBmBac-VP35-VP4; Lane 2, rBmBacmid-VP35-VP4; Lane 3, rBmBac-wild. (C) (Figure S1) Western blotting analysis of rVP35-VP4 expression in BmE cells: Lane M, protein marker; Lane 1, BmE cells infected with rBmBac-VP35-VP4; Lane 2, BmE cells infected with rBmBac-wild; Lane 3, normal BmE cells. (D) (Figure S2) Western blotting analysis of rVP35-VP4 expression in hemolymph of silkworms: Lane M, protein marker; Lane 1, Hemolymph of silkworms infected with rBmBacVP35-VP4; Lane 2, Hemolymph of silkworms infected with rBmBac-wild; Lane 3, normal hemolymph of silkworms.

\subsection{Expression of VP35-VP4 Protein in the BmE Cells and Silkworm upae}

The BmE cells and hemolymph from silkworm infected with P3 baculovirus BmNPVVP35-VP4 were collected after Day 5 and examined by SDS-PAGE and Western blotting. Two protein bands of around 34 and $67 \mathrm{kDa}$ represent the recombinant VP35 and VP4 proteins, respectively, were visualized by Western blotting, suggesting that VP35-VP4 gene was successfully expressed in the BmE cells and silkworm pupae and two proteins achieve complete cleavage via autocatalytic T2A peptide (Figures 2 and 3). The IFA analysis showed that the VP35-VP4 gene was expressed efficiently in the BmE cells (Figure 4).

\subsection{Blood Cell Counting}

Analysis of the hematological parameters indicated that the number of RBCs in the immunized group was $1.96 \pm 0.12 \times 10^{9} / \mathrm{mL}$ and no significant difference compared to the control group. However, the number of WBCs were increased significantly at 14 dpi, significantly higher in immunized fish than in the control group at 14, 21 and $28 \mathrm{dpi}$ $(p<0.05)$ and reached a peak $\left(6.25 \pm 0.81 \times 10^{7} / \mathrm{mL}\right)$ at $21 \mathrm{dpi}(p<0.05)$ (Figure 5A,B). 
A
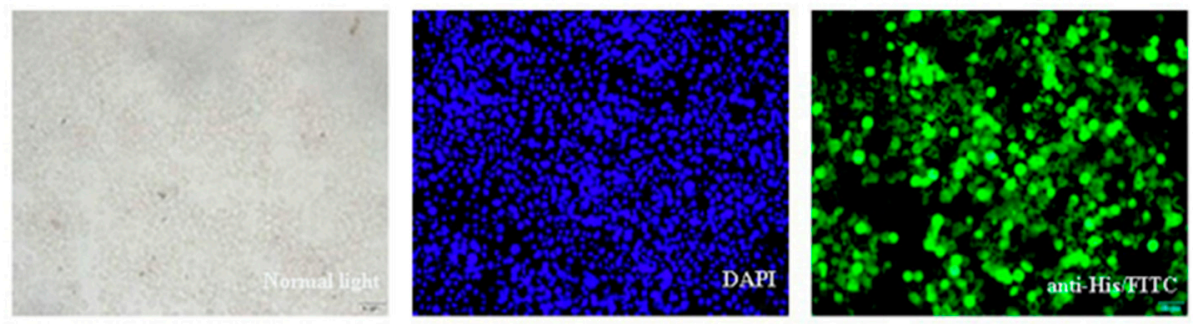

B
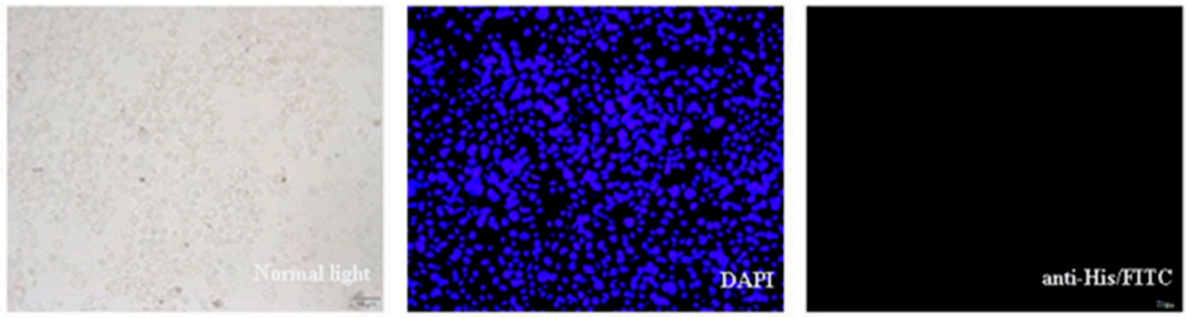

Figure 4. Immunofluorescence assay of recombinant protein expression in BmE cells infected with the recombinant virus: (A) BmE cells infected with rBmBac-VP35-VP4; and (B) BmE cells infected with rBmBac-wild. From left to right: the BmE cells under normal light, UV light and treated with mouse anti-His-tag and Daylight 488-conjugated goat anti-mouse IgG.
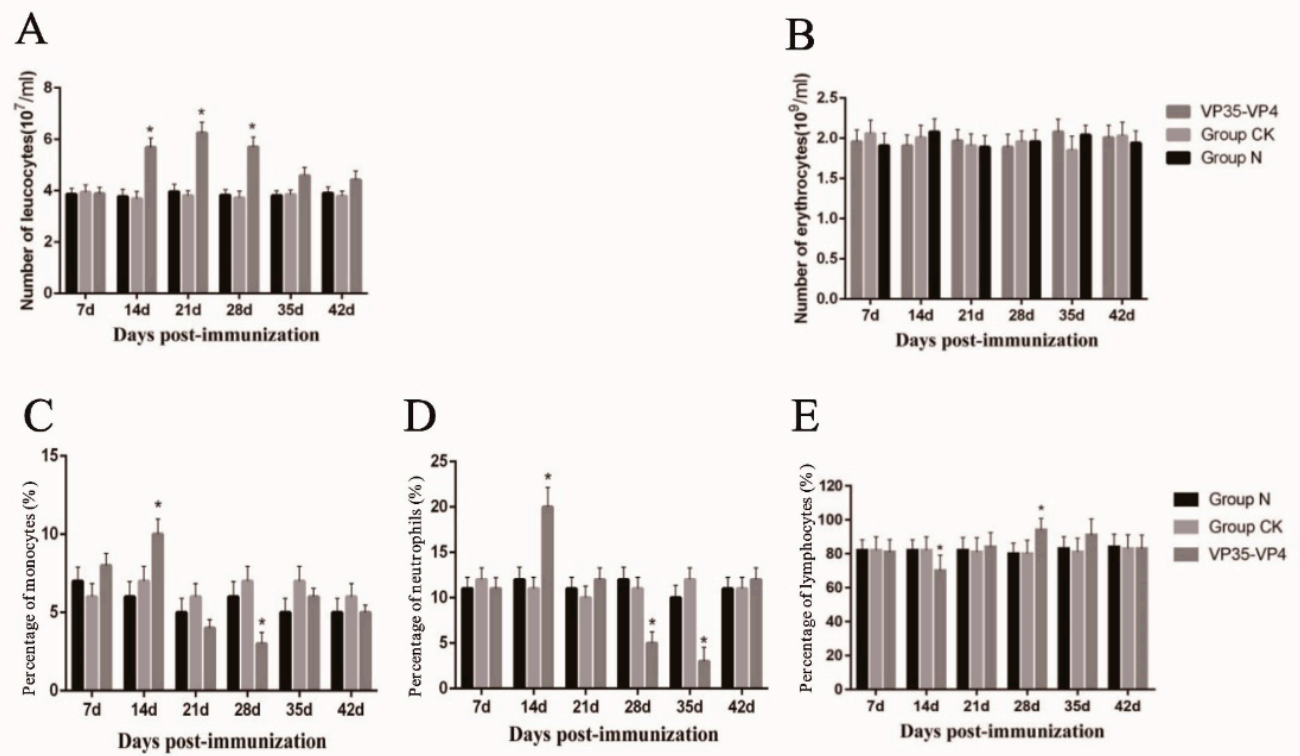

Figure 5. The changes of hematocyte numbers and differential leucocyte count in peripheral blood of grass carp after immunized with oral vaccine. (A-B) The hematocyte number changes of white blood cells and red blood cells in immunized grass carp, respectively. (C-E) Differential leucocyte count changes of monocytes, neutrophils and lymphocytes in immunized grass carp, respectively. * significant difference $(p<0.05)$. The significant difference is compared against the group CK group.

\subsection{Differential Leukocyte Count}

Compared to the control group, the percentage of neutrophils and monocytes among the leukocytes in the immunized group increased significantly and reached a peak $(19.82 \pm 1.18 \%$ and $10.12 \pm 1.18 \%)$ at $14 \mathrm{dpi}(p<0.05)$. The percentage of lymphocyte among the WBCs began to increase and reached the peak of $93.14 \pm 0.82 \%$ on Day $28(p<0.05)$, and it was still higher than the control group at 35 dpi. However, because of the increase in the number of lymphocytes, the percentage of neutrophils and monocytes significantly declined at 28 dpi compared with that of the control group $(p<0.05)$ (Figure $5 \mathrm{C}-\mathrm{E}$ ). 


\subsection{Detection of Serum Antibody Titer by ELISA}

The results of ELISA show that no specific antibody response was observed in the control group and normal group. However, in the immunized group, specific IgM levels increased significantly at Day $14(p<0.01)$ and reached a peak at Day $28(p<0.01)$. Subsequently, the antibody levels decreased steadily, but they were still higher than those of the control group at 35 and $42 \mathrm{dpi}(p<0.05)$ (Figure 6).

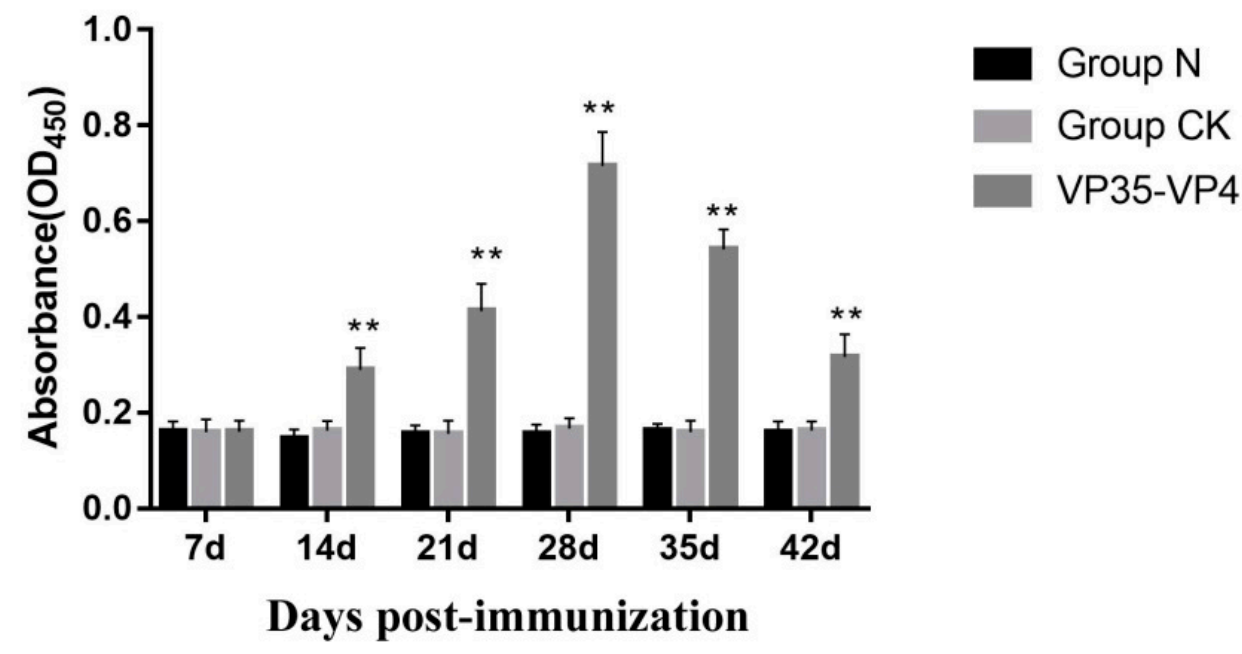

Figure 6. Change of serum IgM antibody levels in grass carp after immunized with oral vaccine. The fish serum was 1:00 dilutions. ${ }^{* *} p<0.01$. The significant difference is compared against the group CK group.

\subsection{Expression of Immune-Related Genes}

The expression levels of immune-related genes IFN-1, TLR22, IL-1 $\beta$, MHC I, Mx and IgM were detected in the liver, spleen, kidney and hindgut by qRT-PCR. The transcript levels of the immune-related genes examined increased significantly with different degrees in the immunized group (VP35-VP4), but no significant differences were detected between the control group (Group CK) and the normal group (Group N) (Figure 7). IFN-1 transcription in the spleen and liver began to increase and reached a peak $(p<0.05)$ at 21 dpi. Significant upregulation was observed at 28,35 and $42 \mathrm{dpi}(p<0.05)$ compared with the control group (Figure 7A). The expression level of the IFN-1 gene in the kidney and hindgut tissue began to increase and reached a peak at 28 and $7 \mathrm{dpi}(p<0.05)$, being approximately 12- and 3-fold higher than that of the control, respectively (Figure 7A). The relative expression levels of TLR22 in all examined tissues started to increase significantly at $14 \mathrm{dpi}(p<0.01)$ and reached their peak values at $21 \mathrm{dpi}(p<0.01), 3-8$ times higher than the control group (Figure 7B). Meanwhile, the mRNA expression of IL-1 $\beta$ and MHC I in liver, spleen, kidney and hindgut was upregulated by $2-5$ times compared to the control group (Figure 7C,D). The mRNA expression levels of Mx in liver, spleen and kidney reached a peak at $21 \mathrm{dpi}(p<0.01)$ (Figure 7E), being approximately 7-, 12- and 25-fold higher than the control, respectively, but there were no significant differences in hindgut. Moreover, in the immunized group, the relative mRNA expression levels of IgM started to increase significantly at $14 \mathrm{dpi}$ in liver and hindgut $(p<0.01)$ and reached the highest value at $21 \mathrm{dpi}$ (Figure 7F), approximately 3.1- and 6.5-fold the control, respectively. In kidney and spleen, the IgM expression levels of the immunized group began to rise significantly at 14 and $21 \mathrm{dpi}$ and reached their peak at $28 \mathrm{dpi}(p<0.01)$, which was about 6-9-fold higher than that in the control group. 

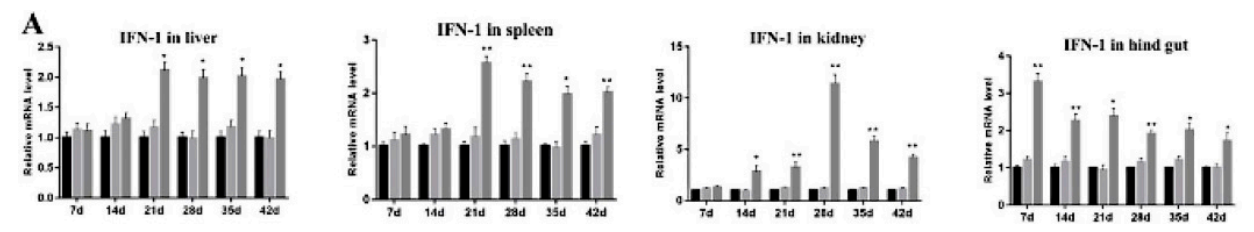

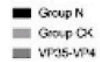
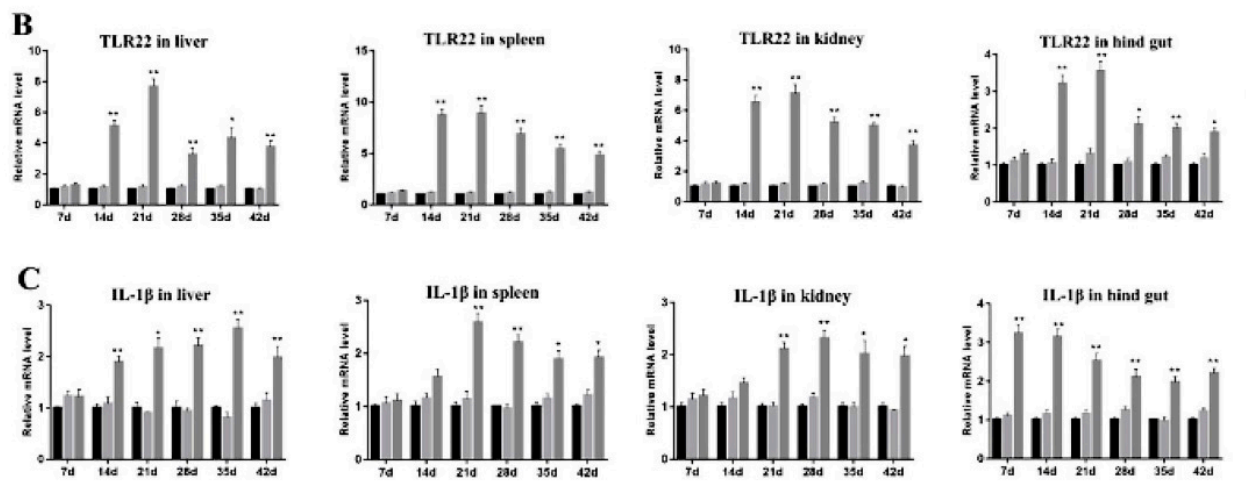

- Goop N

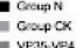

(a)
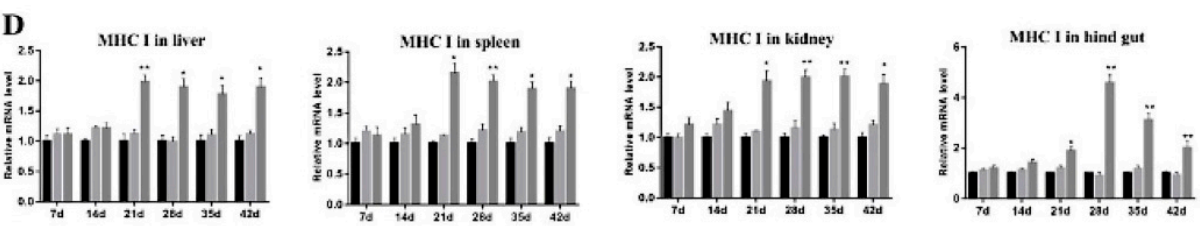

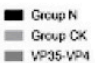
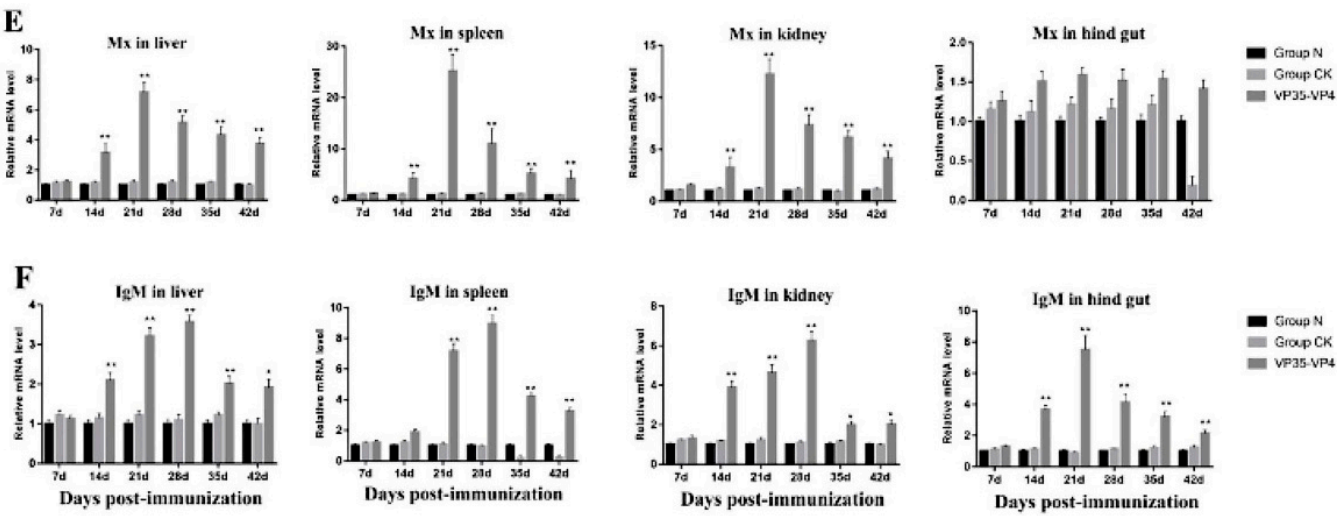

- Group N

Figure 7. qRT-PCR analysis of the expression of immune-related genes in different tissues of the grass carp on Days 7, 14, 21, 28 and 42 after immunization with oral vaccine: (A) IFN-1; (B) TLR22; (C) IL-1 $\beta$; (D) MHC I (E) Mx; and (F) IgM. The mRNA level of each gene was normalized on the basis of $\beta$-actin gene expression. ${ }^{*} p<0.05 ;{ }^{* *} p<0.01$. The significant difference is compared against the group CK group. The relative mRNA expression levels of the immune-related genes were calculated using the $2^{-\Delta \Delta \mathrm{Ct}}$ method.

\subsection{GCRV Challenge Test}

After six weeks of continuous oral immunization, all groups of grass carp were challenged with $1 \times 10^{5} \mathrm{TCID}_{50}$ GCRV at Week 10 to evaluate the protective effect. Mortality and clinical signs of challenge fish were recorded daily for two weeks after challenge. The results show that the dead fish exhibited typical clinical symptoms of GCRV infection, including varying degrees of hemorrhage at operculum, gill, fin base and muscle (Figure 8A), and GCRV was detected by RT-PCR in dead fish. The challenge test showed that the survival percent in the immunized group was $60 \%$ on Day 15 and was significantly higher than that of the normal group $(10 \%)$ and control group $(13 \%)(p<0.01)$ (Figure $8 \mathrm{~B})$. In addition, the immunized group showed a higher relative survival percent in comparison with that of the control group ( $56 \%$ vs. $4 \%, p<0.0001)$. 
A

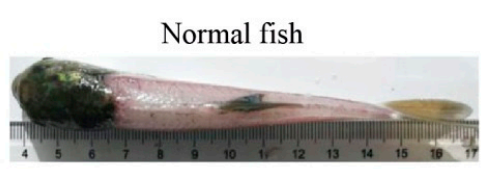

GCRV infected fish

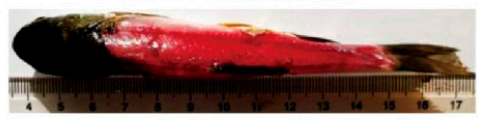

B

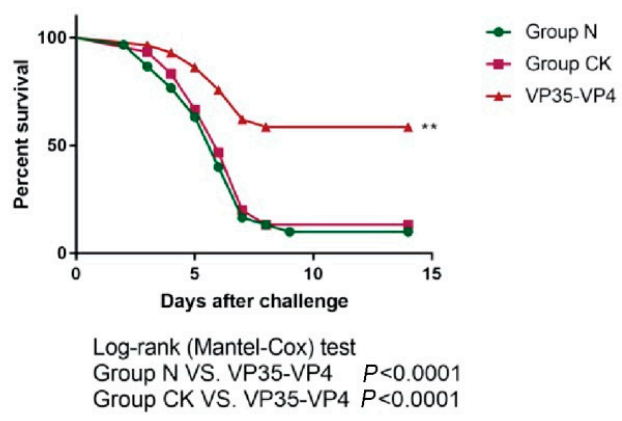

Figure 8. Results of GCRV challenge test. (A) Hemorrhagic symptoms induced by GCRV-106. Images show representative fish specimens in the GCRV-106 and control groups, with the latter exhibiting typical muscular hemorrhagic symptoms. (B) Cumulative survival percentage of the experimental fish challenged with the virus. ${ }^{* *} p<0.01$.

\section{Discussion}

Baculovirus expression system is a rapid and high yield protein production platform, which has been widely used in basic research and the pharmaceutical industry due to its advantages such as high expression, eukaryotic post-translational modifications and self-assembly of the viral capsid protein into virus-like particles (VLPs) [24-26]. Liu et al. used the baculovirus expression system to express the VP6 protein of GCRV and showed that the baculovirus-vectored vaccine can induce immune responses in fish and have the potential to be developed as an oral subunit vaccine [27]. Based on bioinformatic analysis and previous reports, the VP35 and VP4 proteins of GCRV II were predicted to encode the outer capsid proteins and could induce immune response in the fish against GCRV infection $[8,11,12,28]$. In the present study, we used the baculovirus expression system to express the recombinant VP35-VP4 proteins to prepare subunit vaccine and investigated its immune responses and efficacy against GCRV infection in grass carp. In our work, the VP35 and VP4 proteins are linked by self-cut 2A peptide to generate a subunit vaccine construct that expresses two full-length antigens from a single open reading frame. The $2 \mathrm{~A}$ sequence is an oligopeptide of the picornavirus family and can undergo post-translational self-cleavage [29]. The novel "cleavage" event in 2A peptide sequence enables efficient, stoichiometric production of discrete protein products in a single vector and separation of genes placed between $2 \mathrm{~A}$ peptide sequences is nearly $100 \%$ [30]. In this work, two protein bands can be clearly observed at about 34 and $67 \mathrm{kDa}$, representing the recombinant VP35 and VP4 proteins, which suggests that VP35 and VP4 proteins were expressed separately in BmN cells and silkworm by the cleavage action of self-cut T2A peptide and have high shear cut efficiency and balanced upstream and downstream expressions. In addition, a large amount of fluorescence was observed by IFA, indicating that the VP35-VP4 protein was efficiently expressed in BmE cells.

In fish immunization, the route of immunization also plays an important role. Oral vaccines have received increasing attention because they are effective and easy to operate by directly feeding vaccines without causing any stress to the fish $[27,31]$. Oral immunization of grass carp with freeze-dried powder of silkworm pupae containing VP7 protein of GCRV elicited significant immune responses against GCRV [27]. In this study, the protective efficacy in grass carp after oral administration were evaluated based on population of blood cells, IgM antibody titer in the serum, expression levels of immune-related genes and RPS of immunized fish following GCRV challenge.

The number of RBCs showed no significant change compared with the control, whereas the number of WBCs in the peripheral blood of fish immunized increased by 1.4-1.7-fold at 14, 21 and $28 \mathrm{dpi}$. The percentages of neutrophils and monocytes in the WBCs increased and were significantly higher than those of control group at $14 \mathrm{dpi}$, while lymphocytes increased significantly at $28 \mathrm{dpi}$. A similar trend was observed in the Chi- 
nese giant salamander that was injected with a BPL-killed iridovirus vaccine and gibel carp vaccination with $\beta$-propiolactone-inactivated cyprinid herpesvirus 2 [21,22]. These results suggest that the WBCs play a more important role in the immune response. After immunization, the numbers of neutrophils and monocytes in the WBCs first increase, indicating that neutrophils and monocytes play an important role in the early innate immune response, and then the number of lymphocytes increases, which are thought to be involved in a specific immune response mediated by lymphocytes [24].

Serum antibody titer of immunized fish is one of the important indices for the evaluation of a vaccine and reflects the level of immune protection in fish [7]. Previous study showed that the antibody titers increased dramatically at $14 \mathrm{dpi}$ in grass carp immunized with VP4 protein of GCRV-GD108 [3]. In the present study, the results of ELISA indicate that the IgM antibody titer of the immunized group significantly increased on Day 14 and reached the highest level on Day 28, and it was higher than the control group at the sixth week after immunization, consistent with the mRNA expression changes of IgM detected by qRT-PCR, suggesting that the oral vaccine could effectively activate fish humoral immunity and antigens, and the protective effect continued for more than one month.

The thymus, kidney and spleen are the largest lymphoid organs in teleost, and hindgut was the main position for the intake of antigen in grass carp [32-34]. Our results show that the expression levels of TLR22, IFN-1 and Mx were significantly upregulated and peaked at $21 \mathrm{dpi}$ in the liver, spleen and kidney of immunized grass carp. Chen et al. (2018) studied the immune effect of pC-S6 DNA vaccine in grass carp and demonstrated that the mRNA expression levels of IFN-1 and Mx mRNA were significantly upregulated in vaccinated fish [28]. Previous studies showed that the pC-S11 DNA vaccine and recombinant VP35 protein of GCRV could promote induction of IFN-I and TLR22 in grass carp to defend against GCRV infection $[7,24]$. It had been reported that the MHC I and IL-1 $\beta$ expressions were significantly increased in the grass carp immunized by surface displaying BL21/InpN/vp7 vaccine and VP7 DNA vaccine using the bacterial ghost as delivery vehicles against GCRV [2,35]. Our data show that mRNA expression of IL-1 $\beta$ increased significantly in vaccinated grass carp and no significant difference was found in the control group. After 21 days of oral immunization with grass carp, the significant upregulation of MHCI gene was detected, suggesting that oral vaccine could induce the antiviral adaptive immune response in grass carp. IgM is the major immunoglobulin isotype of fish and regarded as an indicator of specific immune responses of teleost fish [36]. In this study, IgM gene expression level was significantly upregulated in oral vaccinated group, which was consistent with the production of specific serum antibodies, indicating that specific immunity was triggered by oral vaccine. Fan et al. (2013) found that grass carp was immunized with DNA vaccine of GCRV VP6, and the IgM mRNA expression levels were significantly upregulated [37]. Therefore, we speculate that the activation of related signaling pathways in immune organs and tissues of immunized fish would be beneficial in resisting GCRV infection [38].

The challenge test showed that the survival rate was $60 \%$ and the relative percent survival was about $56 \%(p<0.01)$ compared to the control CK group. Previous studies have shown that grass carp injected intraperitoneally with $100 \mu \mathrm{L}(0.3 \mathrm{mg} / \mathrm{L})$ recombinant VP35 protein (rVP35) and 1, 3 and $5 \mu \mathrm{g} / \mathrm{g}$ (recombinant protein/fish) of rVP4 have relative percentages of survival of $60 \%, 47 \%$ and $82 \%$ after being challenged with GCRV, respectively $[3,24]$. Although our study appeared to have a similar or lower protective efficacy compared with theirs, the amount of protein $(0.02 \mu \mathrm{g} / \mathrm{g}$ (recombinant protein/fish) $)$ in our study was much lower than those used in previous studies. Jiang et al. (2019) successfully constructed the B. subtilis CotC-VP4 recombinant spores (CotC-VP4 spores), and orally immunized grass carp showed relative percent survival (RPS) of $47 \%$ [37]. In our research, the orally immunized group with the recombinant protein VP35-VP4 showed RPS of 56\%, which is higher than that in previous study, thus we speculate that it might be that the two outer capsid proteins VP35 and VP4 of GCRV could induce a higher immune response than that with a single protein in grass carp. 


\section{Conclusions}

Our results reveal that the baculovirus expression system is very useful to produce the recombinant subunit vaccine VP35-VP4 of GCRV. The recombinant subunit vaccine VP35-VP4 induced not only innate immunity but also humoral and cellular immunity after oral administration in grass carp and provided protection against GCRV infection. In addition, the interaction between VP35 and VP4, as well as other viral proteins, during GCRV invasion need to be further studied.

Supplementary Materials: The following are available online at https:/ /www.mdpi.com/2076-393 X/9/1/41/s1, Figure S1: Figure 3C'S uncropped blots, Figure S2: Figure 3D'S uncropped blotS.

Author Contributions: C.M., Q.Z., V.N.V. and Y.F. conceived and designed the experiments; C.M. and Y.F. performed the experiments; C.M., Q.Z. and Y.F. analyzed the data and wrote the paper; V.N.V. and L.Z. critically discussed results, co-edited paper; L.Z. and Y.F. supplied funds; Y.M.; and Y.Z., N.J., W.L., Y.L. and M.X. revised the paper. All authors have read and agreed to the published version of the manuscript.

Funding: This work was supported by the National Key R\&D Program of China (2019YFD0900101), the National Natural Science Foundation of China (Grant No. 31772894), the State Key Laboratory of Developmental Biology of Freshwater Fish (2017KF004), the Central Public-interest Scientific Institution Basal Research Fund, CAFS (2017GH16, 2020TD44) and the Earmarked Fund for China Agriculture Research System (CARS-46-11).

Institutional Review Board Statement: The experiment was carried out in strict accordance with the Guide for the Care and Use of Laboratory Animals Monitoring Committee of Hubei Province, China, and the internal protocols (No. YFI2019fanyuding-02) were approved by the Committee on the Ethics of Animal Experiments at the Yangtze River Fisheries Research Institute, Chinese Academy of Fishery Sciences.

Informed Consent Statement: Informed consent was obtained from all subjects involved in the study.

Data Availability Statement: The authors confirm that the data supporting the findings of this study are available within the article and its supplementary materials.

Conflicts of Interest: The authors declare no conflict of interest.

\section{References}

1. Rao, Y.; Su, J. Insights into the antiviral immunity against grass carp (Ctenopharyngodon idella) reovirus (GCRV) in grass carp. J. Immunol. Res. 2015, 2015, 670437. [CrossRef] [PubMed]

2. Hao, K.; Chen, X.; Qi, X.; Yu, X.; Du, E.; Ling, F.; Zhu, B.; Wang, G. Protective immunity of grass carp induced by DNA vaccine encoding capsid protein gene (vp7) of grass carp reovirus using bacterial ghost as delivery vehicles. Fish. Shellfish Immunol. 2017, 64, 414-425. [CrossRef] [PubMed]

3. Tian, Y.; Ye, X.; Zhang, L.; Deng, G.; Bai, Y. Development of a novel candidate subunit vaccine against Grass carp reovirus Guangdong strain (GCRV-GD108). Fish. Shellfish Immunol. 2013, 35, 351-356. [CrossRef] [PubMed]

4. Chen, Y.; Jiang, Y. Morphological and physico-chemical characterization of the hemorrhagic virus of grass carp. Sci. Bull. 1984, 6, 832-835.

5. Wang, Q.; Zeng, W.; Liu, C.; Zhang, C.; Wang, Y.; Shi, C.; Wu, S. Complete genome sequence of a reovirus isolated from grass carp, indicating different genotypes of GCRV in China. J. Virol. 2012, 86, 12466. [CrossRef] [PubMed]

6. He, L.; Zhang, A.; Pei, Y.; Chu, P.; Li, Y.; Huang, R.; Liao, L.; Zhu, Z.; Wang, Y. Differences in responses of grass carp to different types of grass carp reovirus (GCRV) and the mechanism of hemorrhage revealed by transcriptome sequencing. BMC Genomics. 2017, 18, 452. [CrossRef]

7. Gao, Y.; Pei, C.; Sun, X.; Zhang, C.; Li, L.; Kong, X. Plasmid pcDNA3.1-s11 constructed based on the $\mathrm{S11}$ segment of grass carp reovirus as DNA vaccine provides immune protection. Vaccine. 2018, 36, 3613-3621. [CrossRef]

8. Liu, H.; Chiou, P.P.; Gong, H.Y.; Chou, H.Y. Cloning of the major capsid protein (MCP) of grouper iridovirus of Taiwan (TGIV) and preliminary evaluation of a recombinant MCP vaccine against TGIV. Int. J. Mol. Sci. 2015, 16, 28647-28656. [CrossRef]

9. Pei, C.; Gao, Y.; Sun, X.; Li, L.; Kong, X. A developed subunit vaccine based on fiber protein VP56 of grass carp reovirus providing immune protection against grass carp hemorrhagic disease. Fish. Shellfish Immunol. 2019, 90, 12-19. [CrossRef]

10. Xue, R.; Liu, L.; Cao, G.; Xu, S.; Li, J.; Zou, Y.; Chen, H.; Gong, C. Oral vaccination of BacFish-vp6 against grass carp reovirus evoking antibody response in grass carp. Fish. Shellfish Immunol. 2013, 34, 348-355. [CrossRef]

11. Yan, X.; Wang, Y.; Xiong, L.; Jian, J.; Wu, Z. Phylogenetic analysis of newly isolated grass carp reovirus. SpringerPlus 2014, 3, 190. [CrossRef] [PubMed] 
12. Su, H.; Liao, Z.; Yuan, G.; Su, J. A plasmid containing CpG ODN as vaccine adjuvant against grass carp reovirus in grass carp Ctenopharyngodon idella. Oncotarget 2017, 8, 86576-86591. [CrossRef] [PubMed]

13. Seng, E.K.; Fang, Q.; Sin, Y.M.; Lam, T.J. Molecular characterization of a major outer capsid protein encoded by the Threadfin aquareovirus (TFV) gene segment 10 (S10). Arch. Virol. 2005, 150, 2021-2036. [CrossRef] [PubMed]

14. Liu, J.; Pei, C.; Gao, X.; Chen, Z.; Zhang, Q. Fish reovirus GCReV-109 VP33 protein elicits protective immunity in rare minnows. Arch. Virol. 2016, 161, 573-582. [CrossRef]

15. Gao, H.; Wang, Y.; Li, N.; Peng, W.; Sun, Y.; Tong, G.; Qiu, H. Efficient gene delivery into mammalian cells mediated by a recombinant baculovirus containing a whispovirus ie1 promoter, a novel shuttle promoter between insect cells and mammalian cells. J. Biotechnol. 2007, 131, 138-143. [CrossRef]

16. Chen, C.; Lin, C.; Chen, G.; Hu, Y. Baculovirus as a gene delivery vector: Recent understandings of molecular alterations in transduced cells and latest applications. Biotechnol. Adv. 2011, 29, 618-631. [CrossRef]

17. Kato, T.; Sugioka, S.; Itagaki, K.; Park, E.Y. Gene transduction in mammalian cells using Bombyx mori nucleopolyhedrovirus assisted by glycoprotein 64 of Autographa californica multiple nucleopolyhedrovirus. Sci. Rep. UK 2016, 6, 32283. [CrossRef]

18. Galindo-Villegas, J.; Mulero, I.; García-Alcazar, A.; Muñoz, I.; Peñalver-Mellado, M.; Streitenberger, S.; Meseguer, J.; Mulero, $\mathrm{V}$. Recombinant $\mathrm{TNF} \alpha$ as oral vaccine adjuvant protects European sea bass against vibriosis: Insights into the role of the CCL25/CCR9 axis. Fish. Shellfish Immunol. 2013, 35, 1260-1271. [CrossRef]

19. Mu, C.; Vakharia, V.N.; Zhou, Y.; Jiang, N.; Liu, W.; Meng, Y.; Li, Y.; Xue, M.; Zhang, J.; Zeng, L.; et al. Novel Subunit Vaccine Based on Outer Capsid Proteins of Grass Carp Reovirus (GCRV) Provides Protective Immunity against GCRV Infection in Rare Minnow (Gobiocypris rarus). Pathogens 2020, 9, E945. [CrossRef]

20. Blaxhall, P.C.; Daisley, K.W. Routine haematological methods for use with fish blood. J. Fish. Biol. 1973, 5, 771-781. [CrossRef]

21. Zhang, L.; Ma, J.; Fan, Y.; Zhou, Y.; Xu, J.; Liu, W.; Gu, Z.; Zeng, L. Immune response and protection in gibel carp, Carassius gibelio, after vaccination with $\beta$-propiolactone inactivated cyprinid herpesvirus 2. Fish. Shellfish Immunol. 2016, 49, 344-350. [CrossRef] [PubMed]

22. Liu, W.; Xu, J.; Ma, J.; LaPatra, S.E.; Meng, Y.; Fan, Y.; Zhou, Y.; Yang, X.; Zeng, L. Immunological responses and protection in Chinese giant salamander Andrias davidianus immunized with inactivated iridovirus. Vet. Microbiol. 2014, 174, 382-390. [CrossRef] [PubMed]

23. Schmittgen, T.D.; Livak, K.J. Analyzing real-time PCR data by the comparative C(T) method. Nat. Protoc. 2008, 3, 1101. [CrossRef] [PubMed]

24. Gao, Y.; Pei, C.; Sun, X.; Zhang, C.; Li, L.; Kong, X. Novel subunit vaccine based on grass carp reovirus VP35 protein provides protective immunity against grass carp hemorrhagic disease. Fish. Shellfish Immunol. 2018, 75, 91-98. [CrossRef]

25. Oers, M.M.; Pijlman, G.P.; Vlak, J.M. Thirty years of baculovirus-insect cell protein expression: From dark horse to mainstream technology. J. Gen. Virol. 2015, 96, 6-23. [CrossRef]

26. Kost, T.A.; Kemp, C.W. Fundamentals of Baculovirus Expression and Applications. Springerplus 2016, 896, 187-197.

27. Liu, B.; Gong, Y.; Li, Z.; Hu, X.; Cao, G.; Xue, R.; Gong, C. Baculovirus-mediated GCRV vp7 and vp6 genes expression in silkworm and grass carp. Mol. Biol. Rep. 2016, 43, 509-515. [CrossRef]

28. Chen, D.; Yao, Y.; Cui, Z.; Zhang, X.; Peng, K.; Guo, X.; Wang, B.; Zhou, Y.; Li, S.; Wu, N.; et al. Comparative study of the immunoprotective effect of two DNA vaccines against grass carp reovirus. Fish. Shellfish Immunol. 2018, 75, 66-73. [CrossRef]

29. Mir, F.A.; Kaufmann, S.H.; Eddine, A.N. A multicistronic DNA vaccine induces significant protection against tuberculosis in mice and offers flexibility in the expressed antigen repertoire. Clin. Vaccine Immunol. 2009, 16, 1467-1475. [CrossRef]

30. Szymczak-Workman, A.L.; Vignali, K.M.; Vignali, D.A. Generation of 2A-linked multicistronic cassettes by recombinant PCR. Cold Spring Harbor. Protocols. 2012, 2012, 251-254. [CrossRef]

31. Zhang, Q.; Xu, B.; Pan, J.; Liu, D.; Lv, R.; Yan, D. Expression and active testing of VP7 from GCRV (Grass carp reovirus) fused with cholera toxin B subunit in rice calli. Protein Expres Purif. 2019, 158, 1-8. [CrossRef] [PubMed]

32. Uribe, C.; Folch, H.; Enríquez, R.; Moran, G. Innate and adaptive immunity in teleost fish: A review. Vet. Med. Czech. 2011, 56, 486-503. [CrossRef]

33. Gomez, D.; Sunyer, J.O.; Salinas, I. The mucosal immune system of fish: The evolution of tolerating commensals while fighting pathogens. Fish. Shellfish Immunol. 2013, 35, 1729-1739. [CrossRef] [PubMed]

34. Siriyappagouder, P.; Galindo-Villegas, J.; Lokesh, J.; Mulero, V.; Fernandes, J.M.O.; Kiron, V. Exposure to Yeast Shapes the Intestinal Bacterial Community Assembly in Zebrafish Larvae. Front. Microbiol. 2018, 9, 1868. [CrossRef]

35. Hao, K.; Chen, X.; Qi, X.; Zhu, B.; Wang, G.; Ling, F. Display of GCRV vp7 protein on the surface of Escherichia coli and its immunoprotective effects in grass carp (Ctenopharyngodon idella). Fish. Shellfish Immunol. 2018, 72, 199-209. [CrossRef]

36. Lorenzen, N.; Lapatra, S.E. Immunity to rhabdoviruses in rainbow trout: The antibody response. Fish. Shellfish Immunol. 1999, 9 , 345-360. [CrossRef]

37. Yan, L.; Guo, H.; Sun, X.; Shao, L.; Fang, Q. Characterization of grass carp reovirus minor core protein VP4. Virol. J. 2012, 9, 89. [CrossRef]

38. Jiang, H.; Bian, Q.; Zeng, W.; Ren, P.; Sun, H.; Lin, H.; Tang, Z.; Zhou, X.; Wang, Q.; Wang, Y. Oral delivery of Bacillus subtilis spores expressing grass carp reovirus VP4 protein produces protection against grass carp reovirus infection. Fish. Shellfish Immunol. 2019, 84, 768-780. [CrossRef] 